\title{
Gas-liquid Flow Pattern Recognition Based on Wavelet Packet Energy Entropy of Vortex-induced Pressure Fluctuation
}

\author{
Zhiqiang $\operatorname{Sun}^{1,2}$, Shuai Shao ${ }^{1,2}$, Hui Gong ${ }^{1,2}$ \\ ${ }^{1}$ School of Energy Science and Engineering, Central South University, Changsha 410083, China \\ ${ }^{2}$ Hunan Key Laboratory of Energy Conservation in Process Industry, Central South University, Changsha 410083, China \\ e-mail: zqsun@csu.edu.cn
}

\begin{abstract}
Here we report a novel flow-pattern map to distinguish the gas-liquid flow patterns in horizontal pipes at ambient temperature and atmospheric pressure. The map is constructed using the coordinate system of wavelet packet energy entropy versus total mass flow rate. The wavelet packet energy entropy is obtained from the coefficients of vortex-induced pressure fluctuation decomposed by the wavelet packet transform. A triangular bluff body perpendicular to the flow direction is employed to generate the pressure fluctuation. Experimental tests confirm the suitability of the wavelet packet energy entropy as an ideal indicator of the gas-liquid flow patterns. The overall identification rate of the map is $\mathbf{9 2 . 8 6 \%}$, which can satisfy most engineering applications. This method provides a simple, practical, and robust solution to the problem of gas-liquid flow pattern recognition.
\end{abstract}

Keywords: Gas-liquid flow pattern, vortex-induced pressure fluctuation, wavelet packet decomposition, energy entropy

\section{INTRODUCTION}

$\mathrm{G}$ AS-LIQUID FLOW, the concurrent flow of gaseous and liquid two-phase mixture, abounds in the processes of industries such as petroleum, power, metallurgy, etc. Due to the highly complex interaction between deformable phases, a variety of interfacial geometric structures occurs in different gas-liquid flow systems. Flow patterns or regimes are defined to describe these interfacial structures by various researchers [1]. For instance, bubble, plug, slug, and annular flows are the four typical flow patterns frequently observed in horizontal pipes under adiabatic conditions. It has been acknowledged that the physical mechanisms controlling the pressure drop and the heat transfer coefficient in gas-liquid mixture are intrinsically related to the flow pattern [2]-[4], and therefore the prediction of flow patterns is an important aspect in gas-liquid flow studies.

Identification of gas-liquid flow patterns is mainly based on experimental observations [5]. On account of its common occurrence and convenient acquisition, pressure fluctuation has been widely used in the characterization of gas-liquid flow patterns [6], [7]. However, pressure fluctuation signals are nonlinear and nonstationary in nature, so it is difficult to extract the flow pattern related features from both time and frequency domains precisely and simultaneously [8], [9]. The unsteady wake behind a bluff body is a source full of fluid flow information [10]. Recent investigations have shown that there exist some close couplings between the vortex-induced pressure fluctuation across a bluff body and the regime of the gas-liquid flow [11], [12]. Different from the single-phase Kármán vortex street, the fluctuation of gas-liquid wake involves not only the vortex shedding induced by the continuous phase, but contains the impingement caused by the disperse phase [13]-[15]. Previous studies reveal that it is possible to discern gasliquid flow patterns by appropriate processing of the vortexinduced pressure fluctuation signals [16].

This paper aims to discern various gas-liquid flow patterns by energy entropies of vortex-induced pressure fluctuation.
Original pressure fluctuations acquired across a bluff body are first decomposed by the wavelet packet transform, and the approximation and detail coefficients are obtained. The energy entropy of the coefficients, named the wavelet packet energy entropy, is introduced to indicate the gas-liquid flow patterns. A flow-pattern map constructed using the wavelet packet energy entropy versus the total mass flow rate is then established. The identification rate of the flow-pattern map is finally verified by experimental tests.

\section{WAVELET PACKET ENERGY ENTROPY}

The wavelet packet transform, an extension of the wavelet transform, employs a rich library of redundant bases with arbitrary time-frequency resolution to decompose a signal in both the approximation space and the detail space. Signal $f(t)$ decomposed by the wavelet packet transform is normally represented as [17]:

$$
\begin{gathered}
f(t)=2^{-1 / 2} \sum_{j} H S_{k}^{j}(i) \mu_{2 n}\left(2^{-j-1} t-i\right) \\
+2^{-1 / 2} \sum_{j} G S_{k}^{j}(i) \mu_{2 n+1}\left(2^{-j-1} t-i\right) \\
\mu_{2 n}(t)=\sqrt{2} \sum_{k} h(k) \mu_{n}(2 t-k) \\
\mu_{2 n+1}(t)=\sqrt{2} \sum_{k} g(k) \mu_{n}(2 t-k)
\end{gathered}
$$

where $\mu_{n}(t)$ is the orthogonal wavelet packet of orthogonalscale function.

From (1), it reveals that the signal can be divided into two parts by the wavelet packet transform adopting the recursive relationships defined by (2) and (3): one is the projection on $\mu_{2 n}\left(2^{-j-1} t-i\right)$, signal passing through the low band filter $H$; the other is the projection on $\mu_{2 n+1}\left(2^{-j-1} t-i\right)$ passing through 
the high band filter $G$. Fig.1. illustrates the procedure of a four-level wavelet packet decomposition that is used in this paper, where $c_{i}$ is the simplified representation of the final coefficient.

Entropy is a measure of irregularities of states, which has been recognized as an ideal parameter for quantifying the ordering of nonstationary signals [18]. The energy entropy based on the wavelet packet decomposition was reported to detect and classify power quality disturbances [19]. Due to the orthogonality of wavelet packet decomposition, the sum of the energy of the coefficients should be equal to the total energy of the original signal. Each wavelet packet coefficient includes a certain band of frequency components; hence its energy forms an energy distribution in the frequency domain. Therefore, in this paper the wavelet packet energy entropy is defined as [20]

$$
S=-\sum_{i=1}^{n} p_{i} \lg p_{i}
$$

where $p_{i}=E_{i} / E$ is the percent of the energy of $c_{i}$ in the whole signal energy $\left(E=\sum E_{i}\right)$.

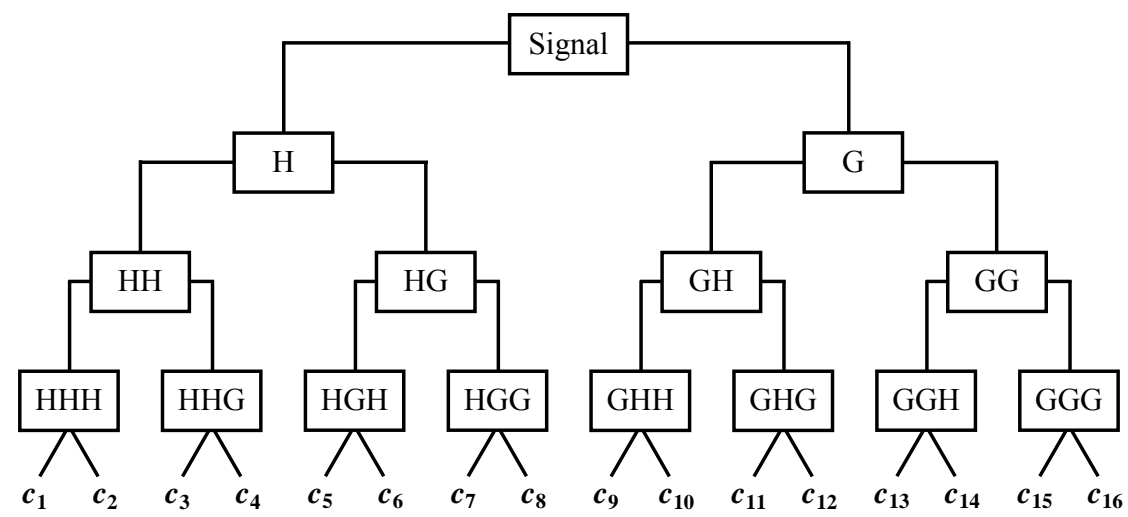

Fig.1. Four-level wavelet packet decomposition tree.

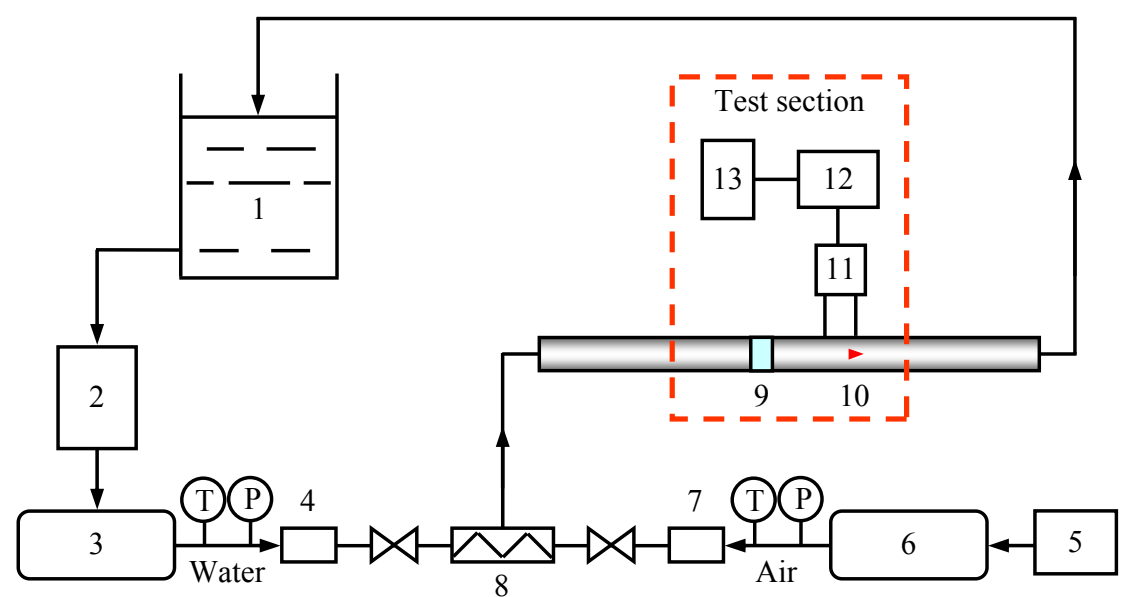

Fig.2. Schematic of the experimental rig: (1) water pool; (2) pump; (3) water surge tank; (4) electromagnetic flowmeter; (5) air compressor; (6) air surge tank; (7) gas flowmeter; (8) static mixer; (9) transparent pipe; (10) bluff body; (11) differential pressure sensor; (12) signal conditioner; (13) digital oscilloscope.

\section{ACQUISITION OF THE VORTEX-INDUCED PRESSURE FLUCTUATIONS}

Experiments were carried out on a gas-liquid two-phase flow test rig by using air and water as the working fluids at ambient temperature and atmospheric pressure, as shown in Fig.2. The compressed air and the pumped water were first pressed into a surge tank to make smooth before their flow rates were measured. The air and water mixed well through a static mixer, then entered into the test section horizontally, and finally recirculated to the water pool. In the test section, a prismatic bluff body perpendicular to the fluid flow direction was mounted in a circular pipe to generate vortex shedding. The inner diameter $D$ of the circular pipe was 50 $\mathrm{mm}$. The cross section of the bluff body was actually a truncated isosceles triangle with the width $w$ of $14 \mathrm{~mm}$, so the blockage ratio of the test section was $b=w / D=0.28$. As accepted by most commercial vortex flowmeters to reduce unwanted fluid noises, this blockage ratio is one of the best choices for flow pattern identification. 

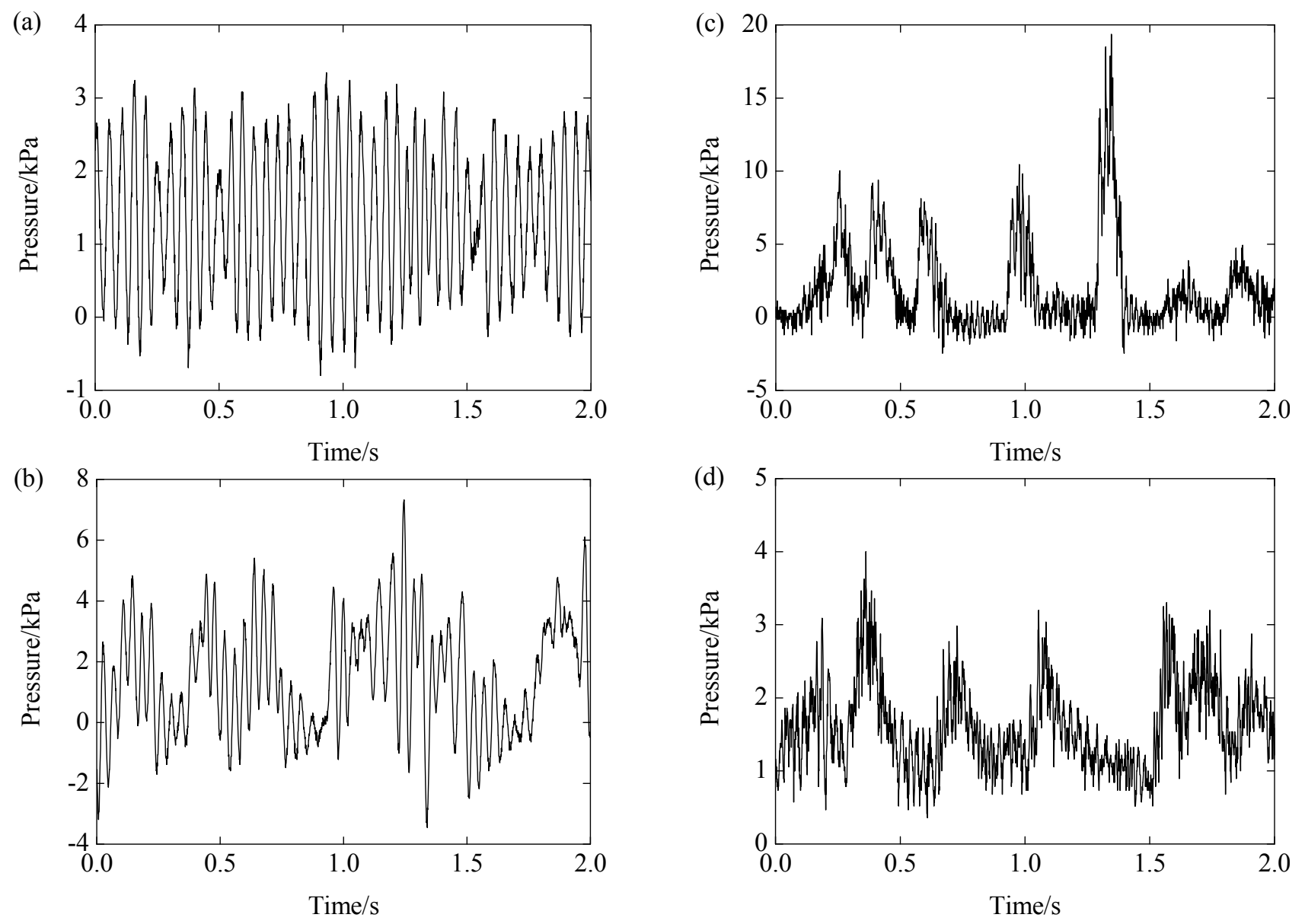

Fig.3. Raw vortex-induced pressure fluctuations in various flow patterns: (a) bubble flow $\left(q_{\mathrm{mT}}=7.92 \mathrm{t} \cdot \mathrm{h}^{-1}, \beta=0.159\right)$; (b) plug flow $\left(q_{\mathrm{mT}}=7.78 \mathrm{t} \cdot \mathrm{h}^{-1}, \beta=0.685\right) ;(\mathrm{c})$ slug flow $\left(q_{\mathrm{mT}}=7.29 \mathrm{t} \cdot \mathrm{h}^{-1}, \beta=0.813\right) ;(\mathrm{d})$ annular flow $\left(q_{\mathrm{mT}}=2.14 \mathrm{t} \cdot \mathrm{h}^{-1}, \beta=0.983\right)$.

Herein the vortex-induced pressure fluctuation signals were detected by the Duct-wall Differential Pressure Method (DDPM) [21]-[24] using a dynamic piezoresistive sensor. The two pressure tappings were located $1.0 \mathrm{D}$ upstream and $0.2 D$ downstream of the bluff body. The fluctuating differential pressure signals were acquired by a fast response digital oscilloscope. A sampling rate of $1 \mathrm{kHz}$ was set throughout the experiments with a $10 \mathrm{~s}$ holding time to obtain all embedded flow information [11]. Thus, each data set contained $10^{4}$ points.

The upstream and downstream straight pipes connecting the bluff body were $70 D$ and $50 D$ long, which ensured that each flow pattern was fully developed in the test section. The flow pattern was recorded manually via a section of a transparent pipe that was installed in front of the bluff body. During the experiments the volumetric flow rates $q_{\mathrm{vG}}$ and $q_{\mathrm{vL}}$ of air and water were $0.1-130 \mathrm{~m}^{3} \cdot \mathrm{h}^{-1}$ with $1.0 \%$ accuracy and $0.5-19 \mathrm{~m}^{3} \cdot \mathrm{h}^{-1}$ with $0.5 \%$ accuracy. The pressure and temperature of air and water were also measured to calculate their densities, so the mass flow rates were obtained by multiplying the volumetric flow rates with their corresponding densities. The total mass flow rate $q_{\mathrm{mT}}$ of the gas-liquid two-phase mixture was $0.648-18.278 \mathrm{t} \cdot \mathrm{h}^{-1}$, and the volumetric void fraction $\beta$ in the test section was 0.017-0.997.
The bubble, plug, slug, and annular flows were observed continually in the experiments. Typical raw vortex-induced pressure fluctuation signals of each flow pattern are shown in Fig.3. It indicates that regular vortex shedding may still occur in the bubble flow, but chaotic oscillations dominate the pressure fluctuations in the other three flow patterns. The differences in these signals provide the feasibility to realize gas-liquid flow pattern recognition; however, it is hard to discern the flow patterns directly from the original signals. So advanced methods for processing and analyzing the pressure fluctuations are needed.

\section{RECOGNITION OF THE GAS-LIQUID FLOW PATTERNS}

The pressure fluctuation signals were decomposed by the four-level wavelet packet. Since it has been used frequently in multiphase flow analysis to decompose time series [25], the four-scale Daubechies base wavelet (db4) was employed in the decomposition. Sixteen wavelet packet coefficients $c_{i}$ were obtained as a result. Fig.4. gives the first three and the last three wavelet packet coefficients of each flow pattern. The sampling frequency $1 \mathrm{kHz}$ is employed throughout the experiments, so the highest frequency that can be acquired from the pressure fluctuation signals is $500 \mathrm{~Hz}$ according to the Shannon's theorem. Table 1. provides the frequency band of each wavelet packet coefficient. 
(a)
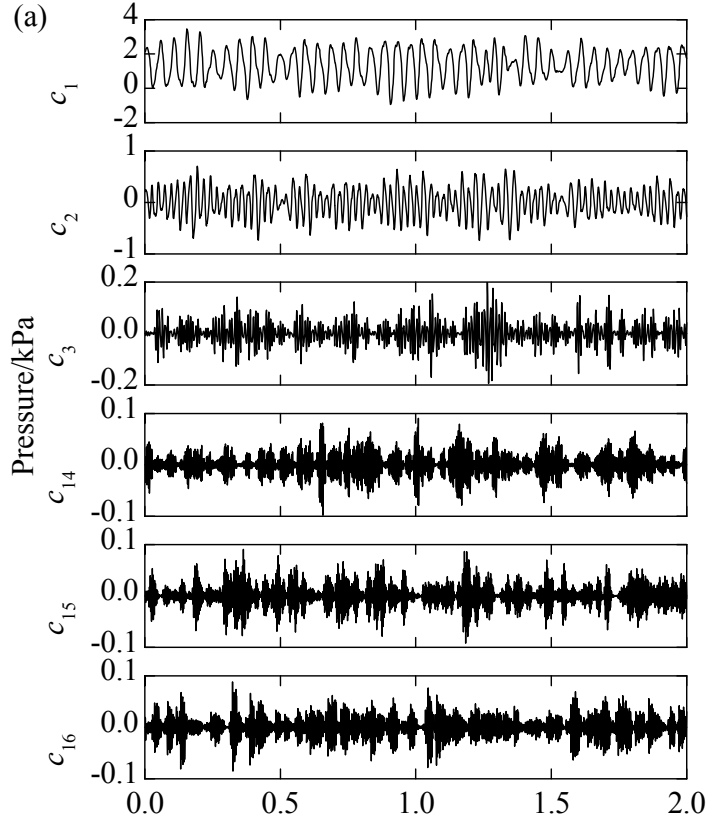

Time/s

(c)
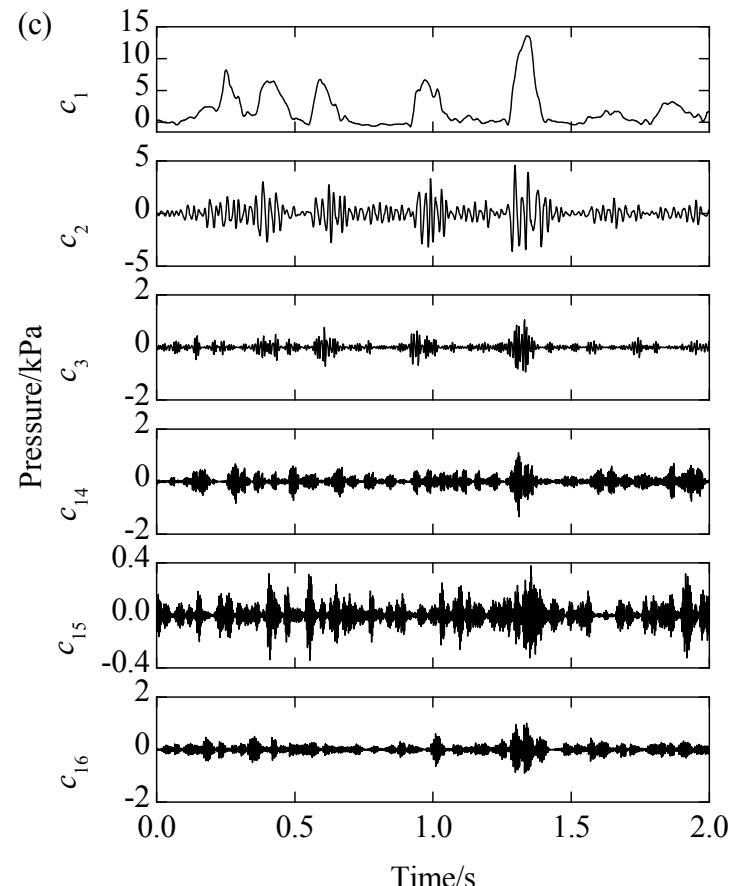

(b)
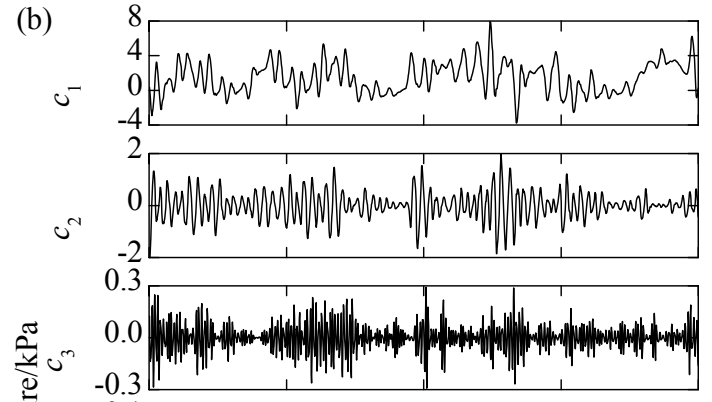

竞
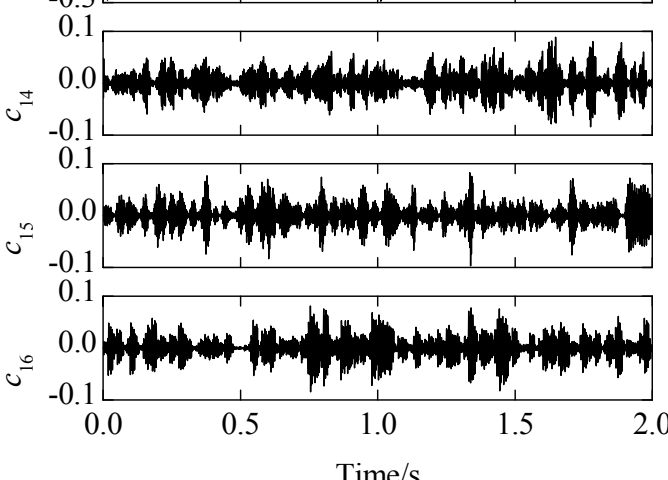

(d)

d)

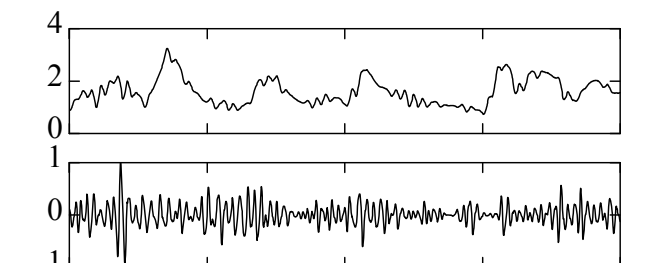

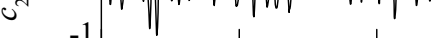
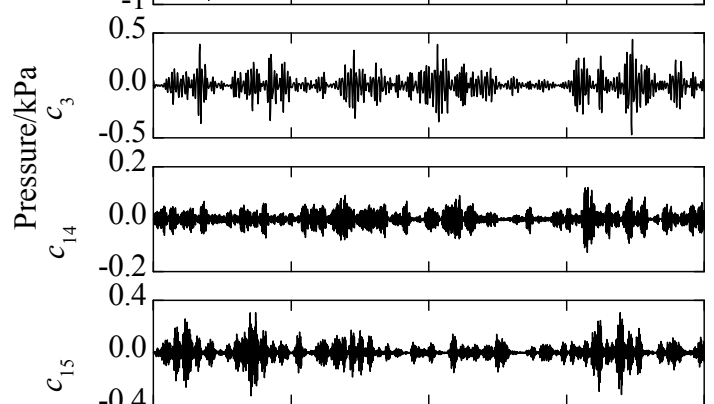

$u^{2}$

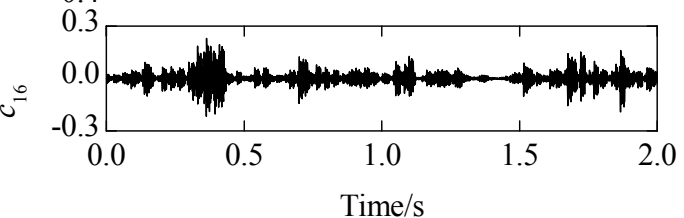

Fig.4. Partial wavelet packet decomposition results of the vortex-induced pressure fluctuations: (a) bubble flow $\left(q_{\mathrm{mT}}=7.92 \mathrm{t} \cdot \mathrm{h}^{-1}, \beta=\right.$ $0.159)$; (b) plug flow $\left(q_{\mathrm{mT}}=7.78 \mathrm{t} \cdot \mathrm{h}^{-1}, \beta=0.685\right)$; (c) slug flow $\left(q_{\mathrm{mT}}=7.29 \mathrm{t} \cdot \mathrm{h}^{-1}, \beta=0.813\right)$; (d) annular flow $\left(q_{\mathrm{mT}}=2.14 \mathrm{t} \cdot \mathrm{h}^{-1}, \beta=\right.$ $0.983)$.

Table 1. Frequency band of the wavelet packet coefficients.

\begin{tabular}{cr||cc}
\hline Coefficient & $\begin{array}{c}\text { Frequency } \\
\text { band/Hz }\end{array}$ & Coefficient & $\begin{array}{c}\text { Frequency } \\
\text { band/Hz }\end{array}$ \\
\hline$c_{1}$ & $0.00-31.25$ & $c_{9}$ & $250.00-281.25$ \\
$c_{2}$ & $31.25-62.50$ & $c_{10}$ & $281.25-312.50$ \\
$c_{3}$ & $62.50-93.75$ & $c_{11}$ & $312.50-343.75$ \\
$c_{4}$ & $93.75-125.00$ & $c_{12}$ & $343.74-375.00$ \\
$c_{5}$ & $125.00-156.25$ & $c_{13}$ & $375.00-406.25$ \\
$c_{6}$ & $156.25-187.50$ & $c_{14}$ & $406.25-437.50$ \\
$c_{7}$ & $187.50-218.75$ & $c_{15}$ & $437.50-468.75$ \\
$c_{8}$ & $218.75-250.00$ & $c_{16}$ & $468.75-500.00$ \\
\hline
\end{tabular}

Table 2. Number of flow patterns.

\begin{tabular}{cccc}
\hline Flow pattern & Total data & Construction data & Test data \\
\hline Bubble & 112 & 95 & 17 \\
Plug & 49 & 42 & 7 \\
Slug & 120 & 102 & 18 \\
Annular & 49 & 42 & 7 \\
Total & 330 & 281 & 49 \\
\hline
\end{tabular}

To construct a flow-pattern map, it is necessary to define an advisable coordinate system that can distinguish various flow patterns fairly. Each wavelet packet coefficient is the 
component of gas-liquid wake fluctuation within a certain band of frequency, so we adopted the wavelet packet energy entropy $S$ as the vertical coordinate of the flow-pattern map. For the horizontal coordinate, we tried to use several flow variables such as the total mass flow rate, the volumetric void fraction, the average density, and the Reynolds number. We find that the total mass flow rate $q_{\mathrm{mT}}$ is a candidate horizontal coordinate according to the $S$ distribution of each flow pattern on the map. The total mass flow rate is comparatively easy to obtain in most cases and remains invariant in the experimental loop, so it is of benefit to the flow pattern identification.
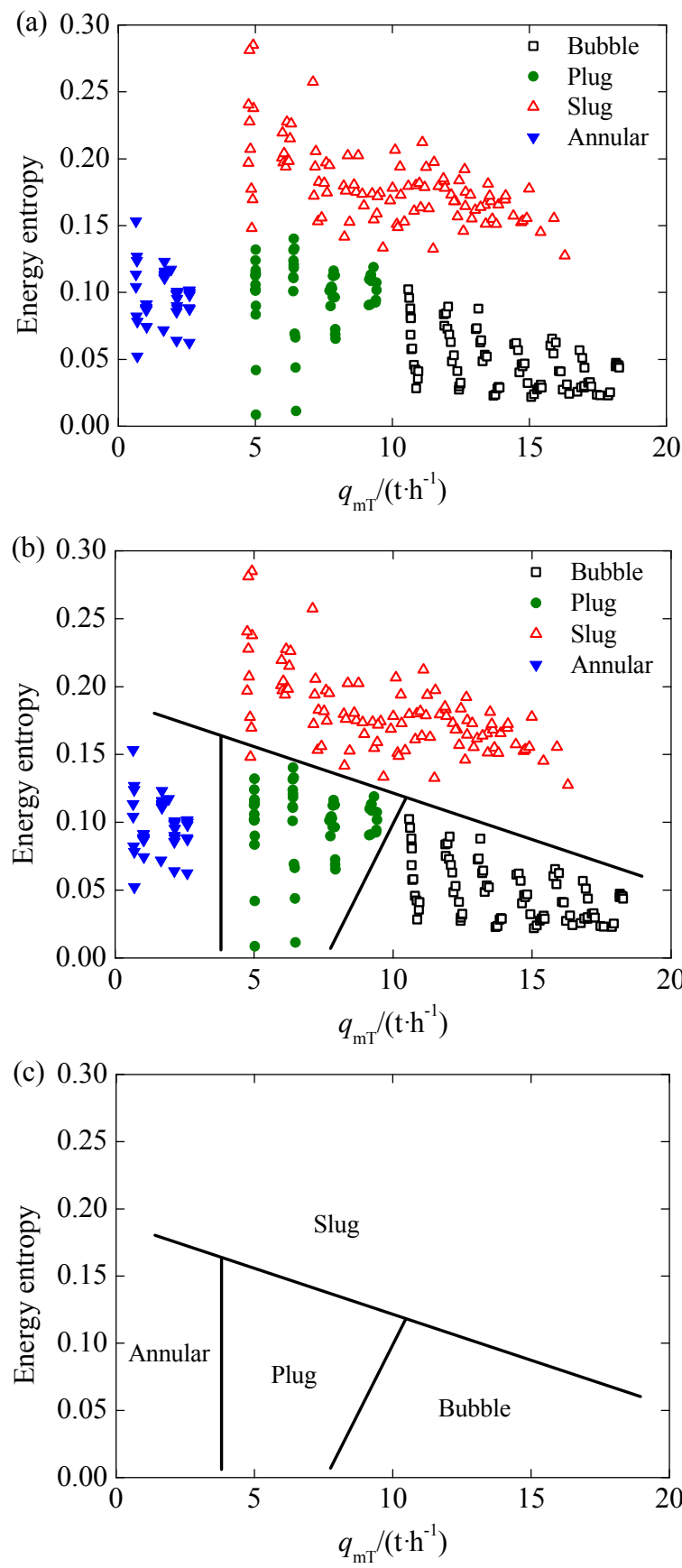

Fig.5. Construction process of the wavelet packet energy entropy based flow-pattern map.

A total of 330 groups of the pressure fluctuation data were acquired during the experiments. The number of each flow pattern recorded is listed in Table 2. Following the common practice of pattern recognition, about $85 \%$ of the total data is selected to construct the flow-pattern maps, which is called the construction data. The remainder of the data is used as the test data for verifying the flow-pattern map.

The construction process of the $S-q_{\mathrm{mT}}$ flow-pattern map, as illustrated in Fig.5., consists of three steps. First of all, the construction data are plotted on a plane with the coordinate system of $S$ versus $q_{\mathrm{mT}}$. The distribution of $S$ in different flow patterns differs with $q_{\mathrm{mT}}$, and these differences provide the rational basis for the discerning of these flow patterns. Second, the zone of each flow pattern is established on the coordinated plane with the construction data. The criterion to delineate the boundaries is that the mid-separate lines are used as far as possible to discern each flow pattern region including both the overlapping and the transitional zones. Third, the construction data are removed, but the boundaries are reserved after the delineation of all the boundaries. Each region on the plane is then tagged with the name of the flow pattern, and the flow-pattern map takes form eventually as Fig.5c.

The test data are plotted on the flow-pattern map devised to examine its validity. It is assumed that, if a point of the test data locates wholly on the region of another flow pattern, it is deemed to be a wrong identification; if a test data point happens to fall on the boundaries, it is regarded as half wrong identification. With this convention, the identification rate of the $S-q_{\mathrm{mT}}$ map is obtained. The overall identification rate of the map developed is $92.86 \%$. Two points of the test data, from the bubble and the slug flows, respectively, fall in the region of the plug flow, one point from the plug flow falls in the region of the bubble flow and another one lies on the boundary, and the other test data are identified successfully.

The above results confirm the suitability of adopting the wavelet packet energy entropy as a rational indicator of the gas-liquid flow pattern. Simultaneously, it reveals that only the neighboring flow patterns are confused on the map. In view of the nature of the complex interaction between phase interfaces, concurrence of multiple flow patterns may exist on the boundary of the near-transition conditions.

\section{CONCLUSIONS}

The gas-liquid flow pattern recognition is achieved by a novel flow-pattern map based on the wavelet packet energy entropy of vortex-induced pressure fluctuation generated by a triangular bluff body perpendicular to the flow direction. Experiments with air and water are conducted at ambient temperature and atmospheric pressure in a 50-mm-diameter horizontal pipe. The pressure fluctuations are recorded in the bubble, plug, slug, and annular flows. The results show that the overall identification rate of the proposed map is $92.86 \%$, and only the neighboring flow patterns are mixed up on the map.

In view of the common occurrence and easy acquisition of pressure fluctuation, this approach is appealing. Moreover, the total mass flow rate is relatively convenient to obtain in most cases and remains invariant throughout the test system. In this regard, the proposed approach is simple, practical and 
robust for engineering applications. However, subjectivity is unavoidable in the delineation of the boundaries between the observed flow patterns. More experimental tests are needed to further increase the identification rate and applicability of the flow-pattern map proposed.

\section{ACKNOWLEDGMENT}

We are grateful for the financial support from the National Natural Science Foundation of China (Grant No. 51006125).

\section{REFERENCES}

[1] Rouhani, S.Z., Sohal, M.S. (1983). Two-phase flow patterns: A review of research results. Progress in Nuclear Energy, 11 (3), 219-259.

[2] Cheng, L., Ribatski, G., Thome, J.R. (2008). Twophase flow patterns and flow-pattern maps: Fundamentals and applications. Applied Mechanics Reviews, 61 (1), 050802.

[3] Sun, Z. (2010). Mass flow measurement of gas-liquid bubble flow with the combined use of a Venturi tube and a vortex flowmeter. Measurement Science and Technology, 21 (5), 055403.

[4] Sun, Z., Zhang, H. (2010). Measurement of the flow rate and volume void fraction of gas-liquid bubble flow using a vortex flow meter. Chemical Engineering Communications, 197 (2), 145-57.

[5] Deng, X., Yang, W.Q. (2012). Fusion research of electrical tomography with other sensors for two-phase flow measurement. Measurement Science Review, 12 (2), 62-67.

[6] Drahos, J., Cermak, J., Selucky, K., Ebner, L. (1987) Characterization of hydrodynamics regimes in horizontal two-phase flow part II: Analysis of wall pressure fluctuations. Chemical Engineering and Processing, 22 (1), 45-52.

[7] Lin, S., Kew, P.A. (2001). Pressure fluctuation and flow regimes of air-water flow in a small tube. Experimental Heat Transfer, 14 (2), 135-144.

[8] Xie, T., Ghiaasiaan, S.M., Karrilab, S. (2004). Artificial neural network approach for flow regime classification in gas-liquid-fiber flows based on frequency domain analysis of pressure signals. Chemical Engineering Science, 59 (11), 2241-2251.

[9] Ding, H., Huang, Z.Y., Song, Z.H., Yan, Y. (2007). Hilbert-Huang transform based signal analysis for the characterization of gas-liquid two-phase flow. Flow Measurement and Instrumentation, 18 (1), 37-46.

[10] Williamson, C.H.K. (1996). Vortex dynamics in the cylinder wake. Annual Review of Fluid Mechanics, 28 (1), 477-539.

[11] Sun, Z., Zhang, H. (2008). Neural networks approach for prediction of gas-liquid two-phase flow pattern based on frequency domain analysis of vortex flowmeter signals. Measurement Science and Technology, 19 (1), 015401.

[12] Sun, Z., Zhang, H. (2009). Application of empirical mode decomposition based energy ratio to vortex flowmeter state diagnosis. Journal of Central South University of Technology, 16 (1), 154-159.
[13] Hulin, J.P., Fierfort, C., Condol, R. (1982). Experimental study of vortex emission behind bluff obstacles in a gas liquid vertical two phase flow. International Journal of Multiphase Flow, 8 (5), 475490.

[14] Inoue, A., Kozawa, Y., Yokosawa, M., Aoki, S. (1986). Studies on two phase cross flow part I: Characteristics around a cylinder. International Journal of Multiphase Flow, 12 (2), 149-167.

[15] Shakouchi, T., Tian, D., Ida, T. (2002). Behavior of vertical upward gas-liquid two-phase flow past obstacle in rectangular channel. JSME International Journal B, 45 (3), 686-693.

[16] Sun, Z., Gong, H. (2012). Classification of gas-liquid flow patterns by the norm entropy of wavelet decomposed pressure fluctuations across a bluff body. Measurement Science and Technology, 23 (12), 125301.

[17] Tan, C., Dong, F., Wu, M.M. (2007). Identification of gas/liquid two-phase flow regime through ERT-based measurement and feature extraction. Flow Measurement and Instrumentation, 18 (5-6), 255-261.

[18] Uyar, M., Yildirim, S., Gencoglu, M.T. (2008). An effective wavelet-based feature extraction method for classification of power quality disturbance signals. Electric Power Systems Research, 78 (10), 1747-1755.

[19] Hu, G., Zhu, F., Ren, Z. (2008). Power quality disturbance identification using wavelet packet energy entropy and weighted support vector machines. Expert Systems with Applications, 35 (1-2), 143-149.

[20] Yang, Y., Yu, D., Cheng, J. (2006). A roller bearing fault diagnosis method based on EMD energy entropy and ANN. Journal of Sound and Vibration, 294 (1-2), 269-277.

[21] Zhang, H., Huang, Y., Sun, Z. (2006). A study of mass flow rate measurement based on the vortex shedding principle. Flow Measurement and Instrumentation, 17 (1), 29-38.

[22] Sun, Z., Zhang, H., Zhou, J. (2007). Investigation of the pressure probe properties as the sensor in the vortex flowmeter. Sensors and Actuators A, 136 (2), 646-655.

[23] Sun, Z., Zhang, H., Zhou, J. (2008). Evaluation of uncertainty in a vortex flowmeter measurement. Measurement, 41 (4), 349-356.

[24] Sun, Z. (2011). Design and performance of the converging-diverging vortex flowmeter. Metrology and Measurement Systems, 18 (1), 129-136.

[25] Shaikh, A., Al-Dahhan, M.H. (2007). A review on flow regime transition in bubble columns. International Journal of Chemical Reactor Engineering, 5 (1), 1-68.

Received September 5, 2012. Accepted April 2, 2013. 